\title{
Signature Quantization in Fading CDMA With Limited Feedback
}

\author{
Wiroonsak Santipach, Member, IEEE
}

\begin{abstract}
In this work, we analyze the performance of a signature quantization scheme for reverse-link Direct Sequence (DS)- Code Division Multiple Access (CDMA). Assuming perfect estimates of the channel and interference covariance, the receiver selects the signature that minimizes interference power or maximizes signal-to-interference plus noise ratio (SINR) for a desired user from a signature codebook. The codebook index corresponding to the optimal signature is then relayed to the user with a finite number of bits via a feedback channel. Here we are interested in the performance of a Random Vector Quantization (RVQ) codebook, which contains independent isotropically distributed vectors. Assuming arbitrary transmit power allocation, we consider additive white Gaussian noise (AWGN) channel first with no fading and subsequently, with multipath fading. We derive the corresponding SINR in a large system limit at the output of matched filter and linear minimum mean squared error (MMSE) receiver. Numerical examples show that the derived large system results give a good approximation to the performance of finite-size system and that the MMSE receiver achieves close to a single-user performance with only one feedback bit per signature element.
\end{abstract}

Index Terms-Random Vector Quantization, large system limit, signature quantization, limited feedback, multipath fading, CDMA.

\section{INTRODUCTION}

User performance in Direct Sequence (DS)- Code Division Multiple Access (CDMA) depends on a signature code, which can be optimized to increase the signal-to-interference plus noise ratio (SINR). Several works in the literature [1]-[7] have investigated a joint transmitter-receiver signature optimization problem and have shown that the performance difference between optimized and random signatures can be substantial. However, adapting the signature increases the complexity and requires knowledge of the channel and interference covariance at both the transmitter and receiver. All aforementioned works assumed that perfect estimates of the channel and interference covariance were available. This assumption, especially at the transmitter, is not practical.

Typically, a receiver estimates channel coefficients and interference covariance from pilot signals during a training period. The accuracy of the estimation increases with the number of available pilots. On the other hand, the transmitter is usually unable to directly estimate the forward channel

This work was jointly supported by Thailand Commission on Higher Education and the Thailand Research Fund under grant MRG5080385. The material in this paper was presented in part at the IEEE International Conference on Communications, Beijing, China, May 19-23, 2008.

The author is with the Department of Electrical Engineering, Faculty of Engineering, Kasetsart University, Chatuchak, Bangkok, 10900 Thailand (email: wiroonsak.s@ku.ac.th). and may obtain channel information from the receiver via a feedback channel. Thus, accuracy of channel information at the transmitter depends on the available feedback rate, which is normally low. In recent years, many researchers [8]-[15] have proposed feedback schemes in which the receiver computes and quantizes the optimal signature and relays the quantized coefficients to the transmitter via a low-rate feedback channel. References [10]-[15] considered multiantenna systems where spatial signatures were optimized and quantized. In the present paper, the focus is on signature quantization in DS-CDMA and its performance, which depends largely on the quantization codebook and available feedback rate.

The signature codebook is known a priori at both the transmitter and receiver. With $B$ feedback bits, the receiver selects the signature vector, which maximizes the instantaneous SINR, from $2^{B}$-signature codebook and relays the corresponding index to the transmitter via an error-free feedback channel. References [8] proposed a Random Vector Quantization (RVQ) codebook, consisting of independent isotropically distributed vectors, and showed that the RVQ codebook was optimal (i.e., maximize the SINR over all codebooks) in a large system limit, in which the number of users $K$, processing gain $N$, and feedback bits $B$, tend to infinity with fixed $\bar{K}=K / N$ and $\bar{B}=B / N$. The upper bound on asymptotic SINR for a single-user matched filter was derived in [8], where in addition, a minimum mean square error (MMSE) receiver was considered and an approximation for a large system SINR was derived. The large system performance was shown to predict the performance of a finite-size system well for small $\bar{B}$.

Recently, [9] derived the exact expression of a large system SINR for the RVQ codebook, assuming that the channel was corrupted by additive white Gaussian noise (AWGN) and the matched filter was used by the receiver. (Similar results for the performance of RVQ in a multiantenna system were derived by [12].) Here we extend those results shown by [9] to a multipath fading channel and to arbitrary transmit power across users. We apply similar techniques to those used in [9], [12] to derive expressions for an asymptotic SINR with a linear MMSE receiver. For the MMSE receiver, we first consider the AWGN channel without fading and derive the exact expression for a large system SINR, which is a function of $\bar{K}$ and $\bar{B}$. We remark that the expressions for the MMSE receiver are not trivial extensions of [9], [12]. A comparison is shown between the large system SINR and the approximation derived in [8], which overestimates the performance for large $\bar{B}$. Numerical examples show that the performance of the finite-size system is estimated very well by the large system results. From the examples shown, a linear MMSE receiver with one feedback 
bit per signature element achieves close to the performance with unlimited feedback.

\section{SYSTEM MODEL}

We consider a discrete-time reverse-link synchronous DSCDMA in which there are $K$ users and processing gain $N$. The $N \times 1$ received vector is given by

$$
\boldsymbol{r}=\sum_{k=1}^{K} \sqrt{A_{k}} \boldsymbol{H}_{k} \boldsymbol{s}_{k} b_{k}+\boldsymbol{n}
$$

where $\sqrt{A_{k}}$ is the amplitude of user $k, \boldsymbol{H}_{k}$ is the $N \times N$ channel matrix for user $k, s_{k}$ is the $N \times 1$ signature vector for user $k, b_{k}$ is the transmitted symbol for user $k$, and $\boldsymbol{n}$ is AWGN with zero mean and covariance $\sigma_{n}^{2} \boldsymbol{I}$. For the AWGN channel with no fading, $\boldsymbol{H}_{k}=\boldsymbol{I}$. For the frequency-selective channel, we assume that the symbol duration is much longer than the delay spread and, thus, we discard any inter-symbol interference. Assuming that each user traverses $L$ Rayleigh fading paths, the channel matrix is given by

$$
\boldsymbol{H}_{k}=\left[\begin{array}{ccccccc}
h_{k, 1} & 0 & \cdots & 0 & 0 & \cdots & 0 \\
\vdots & h_{k, 1} & & \vdots & 0 & & \vdots \\
h_{k, L} & \vdots & \ddots & 0 & \vdots & & 0 \\
0 & h_{k, L} & & h_{k, 1} & 0 & \cdots & 0 \\
\vdots & 0 & \ddots & \vdots & h_{k, 1} & & 0 \\
0 & \vdots & & h_{k, L} & \vdots & \ddots & 0 \\
0 & 0 & \cdots & 0 & h_{k, L} & \cdots & h_{k, 1}
\end{array}\right]
$$

where fading gains for user $k, h_{k, 1}, \ldots, h_{k, L}$, are complex Gaussian random variables with zero means and variances $E\left|h_{k, 1}\right|^{2}, \ldots, E\left|h_{k, l}\right|^{2}$, respectively and are independent across users and fading paths. For a flat fading channel $(L=1), \boldsymbol{H}_{k}=h_{k, 1} \boldsymbol{I}$.

The receiver applies a linear filter on the received signal to obtain the received symbol. We consider both the matched filter and linear MMSE receiver and assume, without loss of generality, that user 1 is the user of interest. The matched filter for user 1 is given by $\boldsymbol{c}_{1}=\tilde{\boldsymbol{s}}_{1}$ where we let $\tilde{\boldsymbol{s}}_{k} \triangleq \boldsymbol{H}_{k} \boldsymbol{s}_{k}$, which is the effective signature for user $k$. The matched filter is simple and can be a performance benchmark for a more complex receiver. The associated SINR is given by

$$
\gamma=\frac{\left|\sqrt{A_{1}} \boldsymbol{c}_{1}^{\dagger} \tilde{\boldsymbol{s}}_{1}\right|^{2}}{\boldsymbol{c}_{1}^{\dagger} \boldsymbol{R}_{1} \boldsymbol{c}_{1}}=\frac{A_{1}\left(\boldsymbol{s}_{1}^{\dagger} \boldsymbol{H}_{1}^{\dagger} \boldsymbol{H}_{1} \boldsymbol{s}_{1}\right)^{2}}{\boldsymbol{s}_{1}^{\dagger} \boldsymbol{H}_{1}^{\dagger} \boldsymbol{R}_{1} \boldsymbol{H}_{1} \boldsymbol{s}_{1}}
$$

where the interference-plus-noise covariance is given by $\boldsymbol{R}_{1}=$ $E\left[\boldsymbol{r}_{1} \boldsymbol{r}_{1}^{\dagger}\right]$ where expectation is over transmitted symbols and noise and

$$
\boldsymbol{r}_{1}=\boldsymbol{r}-\sqrt{A_{1}} \boldsymbol{H}_{1} \boldsymbol{s}_{1} b_{1}=\sum_{k=2}^{K} \sqrt{A_{k}} \boldsymbol{H}_{k} \boldsymbol{s}_{k} b_{k}+\boldsymbol{n} .
$$

Assuming that the $b_{k}$ 's are independent and identically distributed (i.i.d.) with zero mean and unit variance, we have

$$
\boldsymbol{R}_{1}=\sum_{k=2}^{K} A_{k} \tilde{\boldsymbol{s}}_{k} \tilde{\boldsymbol{s}}_{k}^{\dagger}+\sigma_{n}^{2} \boldsymbol{I}=\tilde{\boldsymbol{S}}_{1} \boldsymbol{A}_{1} \tilde{\boldsymbol{S}}_{1}^{\dagger}+\sigma_{n}^{2} \boldsymbol{I},
$$

where $\tilde{\boldsymbol{S}}_{1}$ is the $N \times(K-1)$ effective signature matrix whose columns consist of $\tilde{\boldsymbol{s}}_{k}, \forall k \neq 1$ and $\boldsymbol{A}_{1}$ is the $(K-1) \times(K-1)$ diagonal matrix whose diagonal entries are $A_{2}, \ldots, A_{K}$.

Next, we consider the linear MMSE filter for user 1 given by $\boldsymbol{c}_{1}=\boldsymbol{R}^{-1} \tilde{\boldsymbol{s}}_{1}$ where the received covariance is

$$
\boldsymbol{R}=E\left[\boldsymbol{r} \boldsymbol{r}^{\dagger}\right]=\sum_{k=1}^{K} A_{k} \tilde{\boldsymbol{s}}_{k} \tilde{\boldsymbol{s}}_{k}^{\dagger}+\sigma_{n}^{2} \boldsymbol{I}
$$

with the same assumption that the $b_{k}$ 's are i.i.d. with zero mean and unit variance. Similar to the matched filter, we can compute the SINR for user 1 given by

$$
\beta=A_{1} \boldsymbol{s}_{1}^{\dagger} \boldsymbol{H}_{1}^{\dagger} \boldsymbol{R}_{1}^{-1} \boldsymbol{H}_{1} \boldsymbol{s}_{1}
$$

where the matrix inversion lemma was used to simplify the expression. The linear MMSE receiver is shown to be robust in suppressing multiple-access interference [16]. We note that, for given $\boldsymbol{R}_{1}$ and $\boldsymbol{H}_{1}$, the SINR for user 1 is a function of the signature $s_{1}$ for both receivers.

The receiver, which is assumed to have a perfect estimate of the interference covariance $\boldsymbol{R}_{1}$ and channel matrix $\boldsymbol{H}_{1}$, can optimize the signature for the desired user to maximize the received SINR. Ideally, the receiver sends the optimal signature back to user 1 via a feedback channel and the user changes the signature accordingly. Practically, a feedback channel has limited rate and thus, the receiver can only relay a finite number of feedback bits to the user. (We assume that the feedback does not incur any errors.) With $B$ bits, the receiver selects the signature from a signature set or codebook containing $2^{B}$ signatures. This codebook is designed a priori, and is known to both the user and receiver. The performance of the optimized user depends on the codebook. References [8], [10]-[14], [17] proposed codebook designs and analyzed the associated performance. (All except [8] were in the context of a spatial signature in a multiantenna channel.) In this work, we analyze the performance of a Random Vector Quantization (RVQ) codebook proposed by [8]. We consider an RVQ codebook

$$
\mathcal{V}=\left\{\boldsymbol{v}_{1}, \ldots, \boldsymbol{v}_{2^{B}}\right\}
$$

in which the $\boldsymbol{v}_{j}$ 's are independent isotropically distributed with unit norm $\left(\left\|\boldsymbol{v}_{j}\right\|=1\right)$. In other words, signature vectors in the RVQ codebook are uniformly distributed on a surface of an $N$-dimensional unit sphere. In [8], [9], [12], RVQ was shown to maximize the SINR over all quantization codebooks in a large system limit to be defined. Although RVQ is optimal in the large system limit [8], it was shown to perform close to the optimal codebook designed for a finite-size system [18].

Given the codebook $\mathcal{V}$, the receiver selects

$$
\boldsymbol{s}_{1}=\arg \max _{\boldsymbol{v}_{j} \in \mathcal{V}} \operatorname{SINR}\left(\boldsymbol{v}_{j}\right) \text {. }
$$

The index of the optimal signature vector is relayed to user 1 via a feedback channel without delay. A feedback delay in a time-varying channel will degrade performance, since the optimized signature relayed from the receiver will be outdated by the time it is used at the transmitter. Our model also applies to block fading in which channel coefficients remain relatively static for a period of time to allow meaningful feedback. We 
are interested in analyzing the SINR, which is a function of available feedback bits, for both the matched filter and the MMSE receiver with zero-delay and error-free feedback.

\section{LARge System Performance}

\section{A. Matched filter}

We first consider the AWGN channel without fading $\left(\boldsymbol{H}_{i}=\right.$ $\boldsymbol{I}$, for all $i$ ) for which the optimal signature that maximizes SINR also minimizes the interference. Given the RVQ codebook $\mathcal{V}$, the optimal signature is given by

$$
\boldsymbol{s}_{1}=\arg \min _{\boldsymbol{v}_{j} \in \mathcal{V}}\left\{I\left(\boldsymbol{v}_{j}\right) \triangleq \boldsymbol{v}_{j}^{\dagger} \boldsymbol{R}_{1} \boldsymbol{v}_{j}\right\}
$$

where $I$ is the instantaneous interference power. Since the $\boldsymbol{v}_{j}$ 's in the RVQ codebook are i.i.d., the corresponding $I\left(\boldsymbol{v}_{j}\right)$ 's for a given $\boldsymbol{R}_{1}$ are also i.i.d. and thus, the associated interference averaged over the codebook is given by

$$
\begin{aligned}
E_{\mathcal{V}}[\min & \left.\left\{I\left(\boldsymbol{v}_{1}\right), \ldots, I\left(\boldsymbol{v}_{2^{B}}\right)\right\} \mid \boldsymbol{R}_{1}\right] \\
& =2^{B} \int_{0}^{\infty} x\left[1-G_{I \mid \boldsymbol{R}_{1}}(x)\right]^{2^{B}-1} g_{I \mid \boldsymbol{R}_{1}}(x) \mathrm{d} x
\end{aligned}
$$

where $G_{I \mid \boldsymbol{R}_{1}}(\cdot)$ and $g_{I \mid \boldsymbol{R}_{1}}(\cdot)$ are the cumulative distribution function (cdf) and probability density function (pdf) for $I\left(\boldsymbol{v}_{j}\right)$, respectively. It is difficult to evaluate (11) for any finite $N, K$, and $B$. However, [8], [9] showed that the interference power converges to a deterministic value in a large system limit, in which $K, N$, and $B$ all tend to infinity with fixed normalized load $\bar{K}=K / N$ and normalized feedback bits $\bar{B}=B / N$. Applying the theory of extreme order statistics [19], similar to [8], the large system interference power with a fading channel is given by

$$
I_{\mathrm{rvq}}^{\infty}=\lim _{(N, K, B) \rightarrow \infty} G_{I \mid \boldsymbol{R}_{1}}^{-1}\left(2^{-B}\right)
$$

where the empirical eigenvalue distribution of $\boldsymbol{R}_{1}$ converges almost surely to a nonrandom limit as $(N, K) \rightarrow \infty$ with fixed $K / N$. Rearranging (12) gives

$$
\lim _{\substack{(N, K, B) \rightarrow \infty \\ z \rightarrow I_{\text {rvq }}^{\infty}}}\left[G_{I \mid \boldsymbol{R}_{1}}(z)\right]^{\frac{1}{N}}=2^{-\bar{B}} .
$$

Reference [9, Theorem 1] showed that

$$
\lim _{\substack{(N, K, B) \rightarrow \infty \\ z \rightarrow I_{\text {rvq }}^{\infty}}}\left[G_{I \mid \boldsymbol{R}_{1}}(z)\right]^{\frac{1}{N}}=\exp \left\{-\Psi\left(\rho^{*}, I_{\text {rvq }}^{\infty}\right)\right\}
$$

where

$$
\begin{gathered}
\Psi\left(\rho, I_{\mathrm{rvq}}^{\infty}\right)=\int \log \left(1+\rho\left(\lambda-I_{\mathrm{rvq}}^{\infty}\right)\right) g_{\boldsymbol{R}_{1}}(\lambda) \mathrm{d} \lambda, \\
\rho^{*}=\arg \max _{0<\rho<\frac{1}{I_{\mathrm{rvq}}^{\infty}-\lambda_{\min }}} \Psi\left(\rho, I_{\mathrm{rvq}}^{\infty}\right),
\end{gathered}
$$

and $g_{\boldsymbol{R}_{1}}(\cdot)$ is an asymptotic eigenvalue density for $\boldsymbol{R}_{1}$ and $\lambda_{\min }$ is the asymptotic minimum eigenvalue of $\boldsymbol{R}_{1}$. Equating (13) and (14), we have that $I_{\text {rvq }}^{\infty}$ satisfies

$$
\Psi\left(\rho^{*}, I_{\mathrm{rvq}}^{\infty}\right)=\bar{B} \log (2) .
$$

With (5), Eq. (15) becomes

$$
\begin{aligned}
& \Psi\left(\rho, I_{\text {rvq }}^{\infty}\right) \\
& =\int \log \left(1+\rho\left(\lambda+\sigma_{n}^{2}-I_{\text {rvq }}^{\infty}\right)\right) g_{\boldsymbol{S}_{1} \boldsymbol{A}_{1} \boldsymbol{S}_{1}^{\dagger}}(\lambda) \mathrm{d} \lambda \\
& =\log \left(1+\rho \sigma_{n}^{2}-\rho I_{\text {rvq }}^{\infty}\right)+\int \log (1+\xi \lambda) g_{\boldsymbol{S}_{1} \boldsymbol{A}_{1} \boldsymbol{S}_{1}^{\dagger}}(\lambda) \mathrm{d} \lambda \\
& =\log \left(1+\rho \sigma_{n}^{2}-\rho I_{\mathrm{rvq}}^{\infty}\right)+\nu_{\boldsymbol{S}_{1} \boldsymbol{A}_{1} \boldsymbol{S}_{1}^{\dagger}}(\xi)
\end{aligned}
$$

where

$$
\xi \triangleq \frac{\rho}{1+\rho \sigma_{n}^{2}-\rho I_{\mathrm{rvq}}^{\infty}}
$$

and $\nu_{\boldsymbol{S}_{1} \boldsymbol{A}_{1} \boldsymbol{S}_{1}^{\dagger}}(\cdot)$ is the Shannon transform for an asymptotic eigenvalue distribution for $S_{1} A_{1} S_{1}^{\dagger}$. Reference [20] defined the Shannon transform for a density function $f_{X}(\cdot)$ as follows

$$
\nu_{X}(\gamma)=\int \log (1+\gamma x) f_{X}(x) \mathrm{d} x .
$$

Suppose $s_{k}, 2 \leq k \leq K$, has independent complex Gaussian entries with zero mean and variance $1 / N\left(\left\|\boldsymbol{s}_{k}\right\| \rightarrow 1\right)$. The eigenvalue distribution for $\boldsymbol{S}_{1} \boldsymbol{S}_{1}^{\dagger}$ converges to a deterministic function as $N, K \rightarrow \infty$, with fixed $\bar{K}[21]$ and we assume that the empirical distribution of $A_{2}, \ldots, A_{K}$ converges to a limit. It was shown by [20] that

$$
\begin{array}{r}
\nu_{\boldsymbol{S}_{1} \boldsymbol{A}_{1} \boldsymbol{S}_{1}^{\dagger}}(w)=\bar{K} \nu_{\boldsymbol{A}_{1}}\left(w \eta_{\boldsymbol{S}_{1} \boldsymbol{A}_{1} \boldsymbol{S}_{1}^{\dagger}}(w)\right)-\log \left(\eta_{\boldsymbol{S}_{1} \boldsymbol{A}_{1} \boldsymbol{S}_{1}^{\dagger}}(w)\right) \\
+\eta_{\boldsymbol{S}_{1} \boldsymbol{A}_{1} \boldsymbol{S}_{1}^{\dagger}}(w)-1
\end{array}
$$

where $\eta_{\boldsymbol{S}_{1} \boldsymbol{A}_{1} \boldsymbol{S}_{1}^{\dagger}}(\cdot)$ is the $\eta$-transform for the asymptotic eigenvalue distribution for $\boldsymbol{S}_{1} \boldsymbol{A}_{1} \boldsymbol{S}_{1}^{\dagger}$ and the $\eta$-transform for a distribution for random variable $X$ was defined in [20] as follows

$$
\eta_{X}(\gamma)=\int \frac{1}{1+\gamma x} f_{X}(x) \mathrm{d} x .
$$

With the earlier assumption on the distribution for $\boldsymbol{S}_{1}$, [20] showed that $\eta_{S_{1} \boldsymbol{A}_{1} \boldsymbol{S}_{1}^{\dagger}}(x)$ is the solution to the following fixed point equation

$$
\bar{K}=\frac{1-\eta_{\boldsymbol{S}_{1} \boldsymbol{A}_{1} \boldsymbol{S}_{1}^{\dagger}}(x)}{1-\eta_{\boldsymbol{A}_{1}}\left(x \eta_{\boldsymbol{S}_{1} \boldsymbol{A}_{1} \boldsymbol{S}_{1}^{\dagger}}(x)\right)} .
$$

Combining (17), (20) (23), and (25), we have our first main result.

Theorem 1: The large system interference power $I_{\mathrm{rvq}}^{\infty}$ at the output of single-user matched filter satisfies the following equation

$$
\begin{array}{r}
\max _{0<\rho<\frac{1}{I_{\text {rvq }}^{\infty}-\lambda_{\min }}}\left\{\log \left(1+\rho \sigma_{n}^{2}-\rho I_{\text {rvq }}^{\infty}\right)+\bar{K} \nu_{\boldsymbol{A}_{1}}(\xi(\Theta(\xi))\right. \\
-\log \Theta(\xi)+\Theta(\xi)-1\}=\bar{B} \log (2)
\end{array}
$$

where $\Theta(x)$ is the solution to the following fixed point equation

$$
\bar{K}=\frac{1-\Theta(x)}{1-\eta_{\boldsymbol{A}_{1}}(x \Theta(x))}
$$

and $\xi$ is given by (21). 
Solving for $I_{\text {rvq }}^{\infty}$ requires numerical solution in most cases. However, for equal power allocation $\left(A_{1}=A_{2}=\cdots=A_{K}\right)$, the explicit expression for $I_{\mathrm{rvq}}^{\infty}$ was obtained by [9] as follows.

Corollary 1 (Corollary 1 in [9]): Let

$$
\bar{B}^{*}=\frac{-\bar{K} \log \left(1-\frac{1}{\sqrt{K}}\right)-\sqrt{\bar{K}}}{\log (2)}
$$

for $\bar{K}>1$. For $\bar{K}>1$ and $\bar{B}>\bar{B}^{*}$,

$$
\begin{aligned}
I_{\text {rvq }}^{\infty} & =\sigma_{n}^{2}+(1-\sqrt{\bar{K}})^{2} \\
& +\sqrt{\bar{K}}\left(1-\frac{1}{\sqrt{\bar{K}}}\right)^{1-\bar{K}} \exp (-\sqrt{\bar{K}}-\bar{B} \log (2)) .
\end{aligned}
$$

Otherwise, $I_{\text {rvq }}^{\infty}=Q+\sigma_{n}^{2}$, where $Q$ satisfies the following equation

$$
Q=\bar{K} \mathrm{e}^{(Q-\bar{K}) / \bar{K}} 2^{-\bar{B} / \bar{K}} .
$$

Thus, interference power decreases exponentially with the normalized feedback bits and is near the single-user performance with only a few feedback bits per processing gain. The associated SINR for user 1 in the large system limit is then given by $\gamma_{\mathrm{rvq}}^{\infty}=\frac{1}{I_{\mathrm{rvq}}^{\infty}}$.

If the interfering signatures are orthogonal $\left(\boldsymbol{S}_{1}^{\dagger} \boldsymbol{S}_{1}=\boldsymbol{I}\right)$ with equal power allocation, the eigenvalue distribution for $\bar{K}<1$ is given by

$$
g_{\boldsymbol{S}_{1} \boldsymbol{A}_{1} \boldsymbol{S}_{1}^{\dagger}}(\lambda)=\bar{K} \delta(\lambda-1)+(1-\bar{K}) \delta(\lambda)
$$

where $\delta(\cdot)$ is a Dirac delta function. Evaluating (17) with the distribution in (30), we obtain the following result.

Theorem 2: The large system interference power $I_{\mathrm{rvq}}^{\infty}$ for orthogonal interfering signatures with equal power allocation satisfies the following fixed-point equation

$$
\left(I_{\text {rvq }}^{\infty}-\sigma_{n}^{2}\right)^{\bar{K}}\left(1+\sigma_{n}^{2}-I_{\text {rvq }}^{\infty}\right)^{1-\bar{K}}=\bar{K}^{\bar{K}}(1-\bar{K})^{1-\bar{K}} 2^{-\bar{B}}
$$

for $0<\bar{K}<1$.

For $\bar{K} \approx 1$, we obtain the following approximation

$$
I_{\mathrm{rvq}}^{\infty} \approx \sigma_{n}^{2}+\bar{K} 2^{-\bar{B}} \text {. }
$$

Here, we see clearly that the interference power decreases approximately exponentially with the normalized feedback bits when the system has a heavy load.

For a fading channel, the signal of each user is assumed to propagate $L$ discrete chip-spaced paths with the channel matrix for user $k$ shown in (2). Given the RVQ codebook $\mathcal{V}$, the signature that minimizes the interference power is given by

$$
\boldsymbol{s}_{1}=\arg \min _{\boldsymbol{v}_{j} \in \mathcal{V}}\left\{\tilde{I}\left(\boldsymbol{v}_{j}\right) \triangleq \boldsymbol{v}_{j}^{\dagger} \boldsymbol{H}_{1}^{\dagger} \boldsymbol{R}_{1} \boldsymbol{H} \boldsymbol{v}_{j}\right\} .
$$

We remark that $s_{1}$ may not maximize the SINR in (3). Similar to the channel with no fading, the large system interference power with the RVQ codebook with a fading channel is given by

$$
\tilde{I}_{\mathrm{rvq}}^{\infty}=\lim _{(N, K, B, L) \rightarrow \infty} \min _{\boldsymbol{v}_{j} \in \mathcal{V}} \tilde{I}\left(\boldsymbol{v}_{j}\right),
$$

assuming that a sum of fading variances of each user is finite,

$$
\alpha_{k} \triangleq \sum_{l=1}^{L} E\left|h_{k, l}\right|^{2}<\infty \quad \text { for } \quad 1 \leq k \leq K .
$$

Applying Theorem 11, $\tilde{I}_{\mathrm{rvq}}^{\infty}$ is determined by solving (15) with the asymptotic eigenvalue density $g_{\boldsymbol{R}_{1}}(\cdot)$ replaced with $g_{\boldsymbol{H}_{1}^{\dagger} \boldsymbol{R}_{1} \boldsymbol{H}_{1}}(\cdot)$.

To determine the asymptotic eigenvalue distribution of $\boldsymbol{H}_{1}^{\dagger} \boldsymbol{R}_{1} \boldsymbol{H}_{1}$, we first consider $\boldsymbol{R}_{1} \boldsymbol{H}_{1} \boldsymbol{H}_{1}^{\dagger}$ whose nonzero eigenvalues are the same as those of $\boldsymbol{H}_{1}^{\dagger} \boldsymbol{R}_{1} \boldsymbol{H}_{1}$. Since $\boldsymbol{H}_{1}$ is an $N \times N$ Toeplitz matrix, the $n$th eigenvalue of $\boldsymbol{H}_{1}$ is given by [22]

$$
\lambda_{n}\left(\boldsymbol{H}_{1}\right)=\sum_{l=1}^{L} h_{1, l} \mathrm{e}^{j 2 \pi l \frac{n-1}{N}}
$$

and thus, as $(N, L) \rightarrow \infty$,

$$
\lambda_{n}\left(\boldsymbol{H}_{1}^{\dagger} \boldsymbol{H}_{1}\right)=\left|\sum_{l=1}^{L} h_{1, l} \mathrm{e}^{j 2 \pi l \frac{n-1}{N}}\right|^{2} \longrightarrow \alpha_{1}
$$

almost surely. The limit in (37) follows from the law of large numbers and earlier assumptions that $h_{k, l}$ is independent across a fading path $l$, and $\alpha_{1}<\infty$. Since all eigenvalues of $\boldsymbol{H}_{1}^{\dagger} \boldsymbol{H}_{1}$ converge to the same limit, the asymptotic eigenvalue distribution of $\boldsymbol{H}_{1}^{\dagger} \boldsymbol{R}_{1} \boldsymbol{H}_{1}$ equals that of $\alpha_{1} \boldsymbol{R}_{1}$ where $\boldsymbol{R}_{1}$ is given in (5).

To obtain the $\eta$-transform of $g_{\tilde{S}_{1} \boldsymbol{A}_{1} \tilde{\boldsymbol{S}}_{1}^{\dagger}}(\cdot)$, we solve the following fixed-point equation [20]

$$
\bar{K}=\frac{1-\eta_{\tilde{\boldsymbol{S}}_{1} \boldsymbol{A}_{1} \tilde{\boldsymbol{S}}_{1}^{\dagger}}(x)}{1-\eta_{\tilde{\boldsymbol{A}}_{1}}\left(x \eta_{\tilde{\boldsymbol{S}}_{1} \boldsymbol{A}_{1} \tilde{\boldsymbol{S}}_{1}^{\dagger}}(x)\right)}
$$

where $\eta_{\tilde{\boldsymbol{A}}_{1}}$ is the $\eta$-transform of the asymptotic eigenvalue distribution of

$$
\tilde{\boldsymbol{A}}_{1}=\operatorname{diag}\left\{\alpha_{2} A_{2}, \ldots, \alpha_{K} A_{K}\right\} .
$$

Comparing (38) with (25), we deduce that the asymptotic eigenvalue distributions of $\tilde{\boldsymbol{S}}_{1} \boldsymbol{A}_{1} \tilde{\boldsymbol{S}}_{1}^{\dagger}$ and $\boldsymbol{S}_{1} \tilde{\boldsymbol{A}}_{1} \boldsymbol{S}_{1}^{\dagger}$ are the same. In other words, a multipath interferer is asymptotically equivalent to a single-path interferer with a combined fading gain of $\alpha_{k}$.

Thus, the asymptotic minimum interference power with fading is given by

$$
\tilde{I}_{\mathrm{rvq}}^{\infty}=\alpha_{1} I_{\mathrm{rvq}}^{\infty}
$$

where $I_{\mathrm{rvq}}^{\infty}$ is obtained by Theorem 1 with asymptotic eigenvalue density $g_{S_{1} \tilde{A}_{1} S_{1}^{\dagger}}(\cdot)$ instead. As $N \rightarrow \infty$,

$$
\boldsymbol{s}_{1}^{\dagger} \boldsymbol{H}_{1}^{\dagger} \boldsymbol{H}_{1} \boldsymbol{s}_{1} \rightarrow \alpha_{1} .
$$

Substituting (40) and (41) in (3) gives the associated SINR at the output of the matched filter with a fading channel

$$
\gamma_{\mathrm{rvq}}^{\infty}=\frac{A_{1} \alpha_{1}^{2}}{\tilde{I}_{\mathrm{rvq}}^{\infty}}=\frac{A_{1} \alpha_{1}}{I_{\mathrm{rvq}}^{\infty}} .
$$

\section{B. Linear MMSE Receiver}

The SINR with the optimal signature averaged over the RVQ codebook is given by

$$
\begin{aligned}
& E_{\mathcal{V}}[\max \left.\left\{\beta\left(\boldsymbol{v}_{1}\right), \ldots, \beta\left(\boldsymbol{v}_{2^{B}}\right)\right\} \mid \boldsymbol{R}_{1}, \boldsymbol{H}_{1}\right] \\
&=2^{B} \int_{0}^{\infty} x\left[F_{\beta \mid \boldsymbol{R}_{1}, \boldsymbol{H}_{1}}(x)\right]^{2^{B}-1} f_{\beta \mid \boldsymbol{R}_{1}, \boldsymbol{H}_{1}}(x) \mathrm{d} x
\end{aligned}
$$


where $f_{\beta \mid \boldsymbol{R}_{1}, \boldsymbol{H}_{1}}(\cdot)$ and $F_{\beta \mid \boldsymbol{R}_{1}, \boldsymbol{H}_{1}}(\cdot)$ be pdf and cdf for the output SINR $\beta\left(\boldsymbol{v}_{j}\right)$, respectively. Similar to the matched filter, computing (43) for finite parameters is difficult. Taking the large system limit as $N, K, B \rightarrow \infty$ with fixed ratios, the SINR converges to a deterministic value

$$
\begin{aligned}
\beta_{\mathrm{rvq}}^{\infty} & =\lim _{(N, K, B) \rightarrow \infty} E_{\mathcal{V}}\left[\max \left\{\beta_{1}, \ldots, \beta_{2^{B}}\right\} \mid \boldsymbol{R}_{1}, \boldsymbol{H}_{1}\right] \\
& =\lim _{(N, K, B) \rightarrow \infty} F_{\beta \mid \boldsymbol{R}_{1}, \boldsymbol{H}_{1}}^{-1}\left(1-2^{-B}\right),
\end{aligned}
$$

which can be shown by applying the theory of extreme order statistics [19]. Reference [8] derived the approximation for $\beta_{\text {rvq }}^{\infty}$ by approximating cdf for $\beta\left(\boldsymbol{v}_{j}\right)$ to be Gaussian. The approximation is a function of $\bar{K}, \bar{B}$, and $\sigma_{n}^{2}$ and is good for small $\bar{B}$. For large $\bar{B}$, it overestimates the actual performance. In this section, we derive exact expressions for $\beta_{\mathrm{rvg}}^{\infty}$.

We first consider the ideal channel with no fading $\left(\boldsymbol{H}_{k}=\right.$ $\boldsymbol{I}, \forall k)$. We rearrange (45) to obtain

$$
\lim _{\substack{(N, K, B) \rightarrow \infty \\ z \rightarrow \beta_{\text {rvq }}^{\infty}}}\left[1-F_{\beta \mid R_{1}}(z)\right]^{\frac{1}{N}}=2^{-\bar{B}} .
$$

Similar to [12, eq. (152)] and [9, Theorem 1], it can be shown that

$$
\lim _{\substack{(N, K, B) \rightarrow \infty \\ z \rightarrow \beta_{\text {rvq }}^{\infty}}}\left[1-F_{\beta \mid \boldsymbol{R}_{1}}(z)\right]^{\frac{1}{N}}=\exp \left\{-\Phi\left(\rho^{*}, \beta_{\mathrm{rvq}}^{\infty}\right)\right\}
$$

where

$$
\begin{gathered}
\Phi\left(\rho, \beta_{\mathrm{rvq}}^{\infty}\right)=\int \log \left(1+\rho\left(\beta_{\mathrm{rvq}}^{\infty}-\frac{A_{1}}{\tau+\sigma_{n}^{2}}\right)\right) f_{\boldsymbol{S}_{1} \boldsymbol{A}_{1} \boldsymbol{S}_{1}^{\dagger}}(\tau) \mathrm{d} \tau \\
\rho^{*}=\arg \max _{0<\rho<\frac{1}{\beta_{\text {max }}^{\infty}-\beta_{\mathrm{rvq}}^{\infty}}} \Phi\left(\rho, \beta_{\mathrm{rvq}}^{\infty}\right),
\end{gathered}
$$

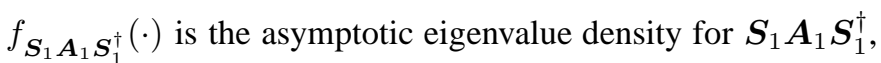
$\boldsymbol{S}_{1}$ is the $N \times(K-1)$ signature matrix whose columns are $s_{2}, \ldots, s_{K}$, and $\beta_{\max }^{\infty}$ is the asymptotic maximum eigenvalue of $A_{1} \boldsymbol{R}_{1}^{-1}$ and corresponds to the SINR with infinite feedback $(\bar{B} \rightarrow \infty)$.

Combining (46) and (47), $\beta_{\mathrm{rvq}}^{\infty}$ satisfies the following fixedpoint equation

$$
\Phi\left(\rho^{*}, \beta_{\mathrm{rvq}}^{\infty}\right)=\bar{B} \log (2) .
$$

To evaluate $\Phi\left(\rho^{*}, \beta_{\mathrm{rvq}}^{\infty}\right)$, we rewrite (48) as follows

$$
\begin{aligned}
& \Phi\left(\rho, \beta_{\text {rvq }}^{\infty}\right) \\
& =\log \left(1+\rho\left(\beta_{\mathrm{rvq}}^{\infty}-\frac{A_{1}}{\sigma_{n}^{2}}\right)\right)+\int \log (1+\zeta \tau) f_{\boldsymbol{S}_{1} \boldsymbol{A}_{1} \boldsymbol{S}_{1}^{\dagger}}(\tau) \mathrm{d} \tau \\
& -\int \log \left(1+\frac{1}{\sigma_{n}^{2}} \tau\right) f_{\boldsymbol{S}_{1} \boldsymbol{A}_{1} \boldsymbol{S}_{1}^{\dagger}}(\tau) \mathrm{d} \tau \\
& =\nu_{\boldsymbol{S}_{1} \boldsymbol{A}_{1} \boldsymbol{S}_{1}^{\dagger}}(\zeta)-\nu_{\boldsymbol{S}_{1} \boldsymbol{A}_{1} \boldsymbol{S}_{1}^{\dagger}}\left(1 / \sigma_{n}^{2}\right)+\log \left(1+\rho\left(\beta_{\mathrm{rvq}}^{\infty}-\frac{A_{1}}{\sigma_{n}^{2}}\right)\right)
\end{aligned}
$$

where

$$
\zeta \triangleq \frac{1+\rho \beta_{\mathrm{rvq}}^{\infty}}{\sigma_{n}^{2}+\rho \beta_{\mathrm{rvq}}^{\infty} \sigma_{n}^{2}-\rho A_{1}}
$$

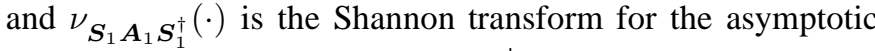
eigenvalue distribution for $\boldsymbol{S}_{1} \boldsymbol{A}_{1} \boldsymbol{S}_{1}^{\dagger}$. With similar steps used to derive Theorem 1, we obtain the following theorem.
Theorem 3: For $\bar{B}$, the large system SINR $\beta_{\mathrm{rvq}}^{\infty}$ is given by

$$
\begin{gathered}
\max _{0<\rho<\frac{1}{\beta_{\max }^{\infty}-\beta_{\text {rq }}^{\infty}}}\left\{\log \left(1+\rho\left(\beta_{\text {rvq }}^{\infty}-\frac{A_{1}}{\sigma_{n}^{2}}\right)\right)+\bar{K} \nu_{\boldsymbol{A}_{1}}(\zeta \Theta(\zeta))\right. \\
-\bar{K} \nu_{\boldsymbol{A}_{1}}\left(\sigma_{n}^{-2} \Theta\left(\sigma_{n}^{-2}\right)\right)-\log (\Theta(\zeta))+\log \left(\Theta\left(\sigma_{n}^{-2}\right)\right) \\
\left.+\Theta(\zeta)-\Theta\left(\sigma_{n}^{-2}\right)\right\}=\bar{B} \log (2)
\end{gathered}
$$

where $\zeta$ and $\Theta(x)$ are given by (54) and (27), respectively.

For an equal-power $\left(A_{1}=A_{2}=\ldots=A_{K}\right)$ system, we can simplify the expression for $\beta_{\mathrm{rvq}}^{\infty}$ as follows.

Corollary 2: We assume no fading, i.i.d. interfering signatures, and equal transmitted power across users. For $\bar{K} \leq 1$, $\beta_{\text {rvq }}^{\infty}$ satisfies the following equation

$$
\begin{aligned}
\log \left(\frac{\bar{K}}{1-\beta_{\text {rvq }}^{\infty} \sigma_{n}^{2}}-\frac{1}{\beta_{\text {rvq }}^{\infty}}\right)+(1-\bar{K}) \log \left(\frac{p}{\sigma_{n}^{2}}\right) \\
+\bar{K} \log \left(\frac{w(p)}{w\left(\sigma_{n}^{2}\right)}\right)-(1-\bar{K}) \log \left(\frac{1-v(p)}{1-v\left(\sigma_{n}^{2}\right)}\right) \\
-v(p)+v\left(\sigma_{n}^{2}\right)=\bar{B} \log (2)
\end{aligned}
$$

where

$$
\begin{aligned}
& w(x)=\frac{1}{2}\left(1+\bar{K}+x+\sqrt{(1+\bar{K}+x)^{2}-4 \bar{K}}\right) \\
& v(x)=\frac{1}{2}\left(1+\bar{K}+x-\sqrt{(1+\bar{K}+x)^{2}-4 \bar{K}}\right)
\end{aligned}
$$

and

$$
p=\frac{1-\beta_{\mathrm{rvq}}^{\infty} \sigma_{n}^{2}}{\bar{K} \beta_{\mathrm{rvq}}^{\infty}-1+\beta_{\mathrm{rvq}}^{\infty} \sigma_{n}^{2}}-\frac{1}{\beta_{\mathrm{rvq}}^{\infty}}+\sigma_{n}^{2} .
$$

For $\bar{K}>1$ and $\bar{B} \leq \bar{B}^{*}, \beta_{\mathrm{rvq}}^{\infty}$ satisfies the following equation

$$
\begin{aligned}
& \log \left(\frac{\bar{K}}{1-\beta_{\text {rvq }}^{\infty} \sigma_{n}^{2}}-\frac{1}{\beta_{\text {rvq }}^{\infty}}\right)+\log \left(\frac{w(p)}{w\left(\sigma_{n}^{2}\right)}\right) \\
& -(\bar{K}-1) \log \left(\frac{\bar{K}-v(p)}{\bar{K}-v\left(\sigma_{n}^{2}\right)}\right)-v(p)+v\left(\sigma_{n}^{2}\right)=\bar{B} \log (2)
\end{aligned}
$$

where

$$
\begin{aligned}
\bar{B}^{*}=\frac{1}{\log (2)}\left(\log \left(\bar{K}-\sqrt{\bar{K}}+\sigma_{n}^{2}\right)+\bar{K} \log (\sqrt{\bar{K}})\right. \\
-\bar{K} \log (\sqrt{\bar{K}}-1)-\sqrt{\bar{K}}-\log \left(w\left(\sigma_{n}^{2}\right)\right) \\
\left.+(\bar{K}-1) \log \left(1-\frac{v\left(\sigma_{n}^{2}\right)}{\bar{K}}\right)+v\left(\sigma_{n}^{2}\right)\right) .
\end{aligned}
$$

For $\bar{K}>1$ and $\bar{B}>\bar{B}^{*}$,

$$
\begin{aligned}
& \beta_{\text {rvq }}^{\infty}= \beta_{\max }^{\infty}\left(1-2^{-\bar{B}}\left[\operatorname { e x p } \left\{\frac{1}{2} \bar{K} \log (\bar{K})\right.\right.\right. \\
&-(\bar{K}-1) \log \left(\frac{\bar{K} \sqrt{\bar{K}}-\bar{K}}{\bar{K}-v\left(\sigma_{n}^{2}\right)}\right)-\log \left(w\left(\sigma_{n}^{2}\right)\right) \\
&\left.\left.\left.\quad+v\left(\sigma_{n}^{2}\right)-\sqrt{\bar{K}}\right\}\right]\right) .
\end{aligned}
$$

The proof is shown in the Appendix.

We can also derive the SINR when the interfering signatures are orthogonal. Substituting the corresponding eigenvalue distribution (30) into (52) and simplifying give the following result. 
Theorem 4: For an orthogonal set of interfering signatures with $0<\bar{K}<1$ and equal power allocation, the large system SINR $\beta_{\text {rvq }}^{\infty}$ is the solution of the following fixed-point equation

$$
\begin{aligned}
&\left(A_{1}-\beta_{\mathrm{rvq}}^{\infty} \sigma_{n}^{2}\right)^{\bar{K}}\left(\beta_{\mathrm{rvq}}^{\infty}-\left(A_{1}-\beta_{\mathrm{rvq}}^{\infty} \sigma_{n}^{2}\right)\right)^{1-\bar{K}} \\
&=\left(\frac{A_{1} \bar{K}}{1+\sigma_{n}^{2}}\right)^{\bar{K}}\left(\frac{A_{1}(1-\bar{K})}{\sigma_{n}^{2}}\right)^{1-\bar{K}} 2^{-\bar{B}} .
\end{aligned}
$$

For a system with heavy load $(\bar{K} \approx 1)$, we have

$$
\beta_{\mathrm{rvq}}^{\infty} \approx \frac{A_{1}}{\sigma_{n}^{2}}-\frac{A_{1} \bar{K}}{\sigma_{n}^{2}\left(1+\sigma_{n}^{2}\right)} 2^{-\bar{B}} .
$$

The first term on the right-hand side of (64) is the singleuser performance and thus, the performance with the MMSE receiver also increases exponentially with $\bar{B}$, which is the same as performance with the matched filter.

Similar to the matched-filter case, a multipath fading is asymptotically equivalent to a single-path fading with combined gain of $\alpha_{k}<\infty$. The SINR with fading channel can be obtained from Theorem 3 , in which we replace $A_{1}$ with $\alpha_{1} A_{1}$, and $\boldsymbol{A}_{1}$ with $\tilde{\boldsymbol{A}}_{1}$.

\section{NUMERICAL RESULTS}

Fig. 1 shows the asymptotic SINR for the MMSE receiver in Corollary 2 versus a normalized feedback bit $\bar{B}$ with different normalized loads $\bar{K}=0.25,0.5,1,1.25$. As expected, the SINR increases with normalized feedback and decreases with normalized load. For $\bar{K}=0.25$, RVQ achieves close to the single-user performance with approximately $\bar{B}=0.5$ ( 0.5 bits per processing gain or degree of freedom). As the number of interfering users increases, the amount of feedback required also increases to achieve a target SINR. For example, $\bar{B}=3$ is needed for a system with $\bar{K}=1$ to achieve close to the singleuser performance. We also compare the asymptotic results with simulation results marked by the plus signs in Fig. 1 We note that the large system results predict the performance of finite-size systems $(N=12)$ well. As $N$ increases, the gap between the simulation and analytical results is expected to close. The RVQ codebook requires an exhaustive search to locate the optimal signature. The search complexity increases exponentially with feedback bits $B$. (For $\bar{B}=3$, the number of entries in the RVQ codebook is $2^{36}$.) Thus, we do not have simulation results for a large $B$.

Fig. 2] shows the large system performance of both receivers, which is obtained from Theorems 1, 4, with different distributions for interfering signatures. We consider both sets of orthogonal and i.i.d. Gaussian interfering signatures. From Fig. 2, the system with independent Gaussian signatures performs a little better than that with orthogonal signatures for both linear receivers. The difference is more pronounced with the matched filter. Which distribution for interfering signatures gives the maximum performance is an interesting open problem.

In Fig. 3. we compare the asymptotic SINR for the MMSE receiver in Corollary 2 with the approximation derived in [8] for $\bar{K}=0.75$ and $\mathrm{SNR}=10 \mathrm{~dB}$. Also shown are the simulation results with $N=12$. The large system SINR is

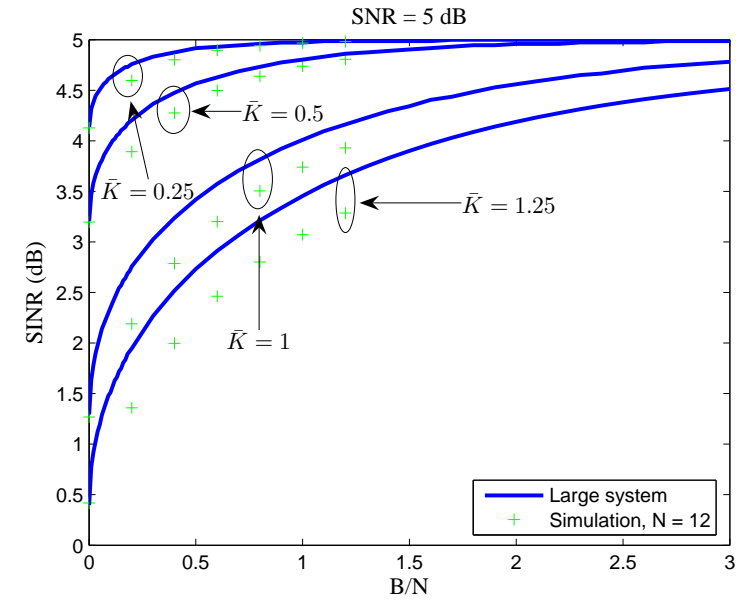

Fig. 1. Large system SINR for MMSE receiver versus normalized feedback bit $\bar{B}$ with different normalized loads $\bar{K}=0.25,0.5,1,1.25$ and SNR $=$ $5 \mathrm{~dB}$.

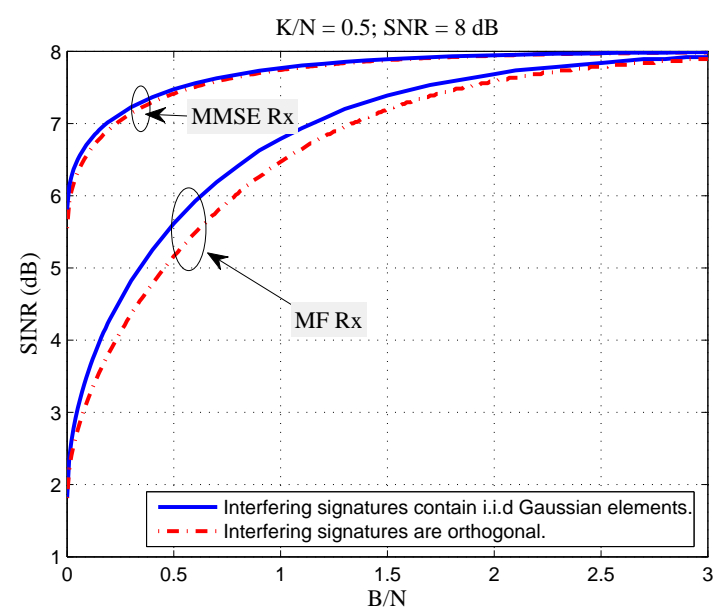

Fig. 2. Large system SINR's for different distributions of interfering signatures with $\bar{K}=0.5$ and $\mathrm{SNR}=8 \mathrm{~dB}$.

closer to the simulated performance than the approximation. We also show the RVQ performance of the matched filter in Corollary 1 [9] with that of the MMSE receiver. The performance difference can be substantial for small to moderate $\bar{B}$. With 1 feedback bit per degree of freedom, the MMSE receiver outperforms a matched filter by as much as $30 \%$. However, the MMSE filter is more complex than the matched filter. Therefore, there is a performance tradeoff between the feedback and receiver complexity.

We also simulated a multipath fading channel, in which each user's signal traverses two paths with different gains $\left(E\left|h_{k, 1}\right|^{2}=0.9\right.$ and $\left.E\left|h_{k, 2}\right|^{2}=0.1, \forall k\right)$. Furthermore, $K$ interfering users are divided into two groups. $K_{1}$ users transmit signal with $A_{k}=P_{1}$ while $K_{2}$ users transmit with $A_{k}=P_{2}$. This scenario may follow from a system with differentiated quality of service. We obtain the large system SINR from Theorem 3 with the asymptotic distribution of $\boldsymbol{A}_{1}$

$$
f_{\boldsymbol{A}_{1}}(a)=\frac{\bar{K}_{1}}{\bar{K}} \delta\left(a-P_{1}\right)+\frac{\bar{K}_{2}}{\bar{K}} \delta\left(a-P_{2}\right)
$$




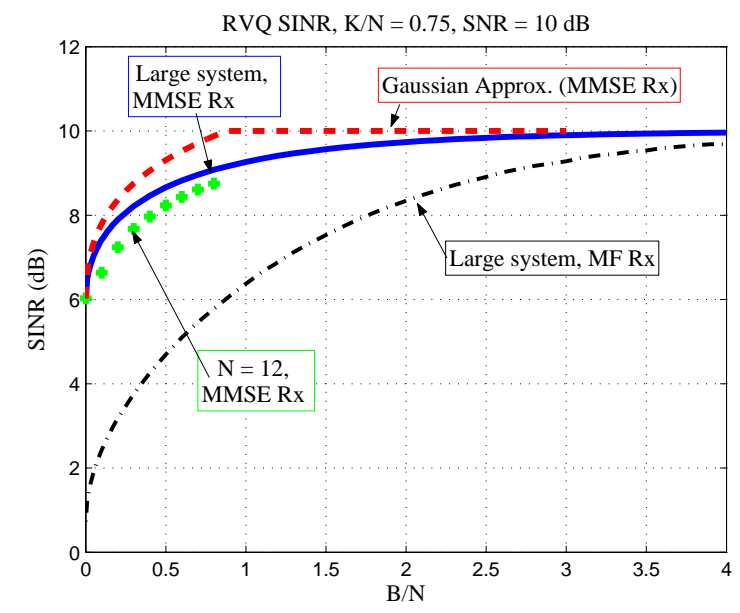

Fig. 3. Large system SINR for MMSE receiver compared with the approximation derived in [8] and the large system SINR for a matched filter [9]. Also shown is the simulation result for $N=12, \bar{K}=0.75$ and $\mathrm{SNR}=10 \mathrm{~dB}$.

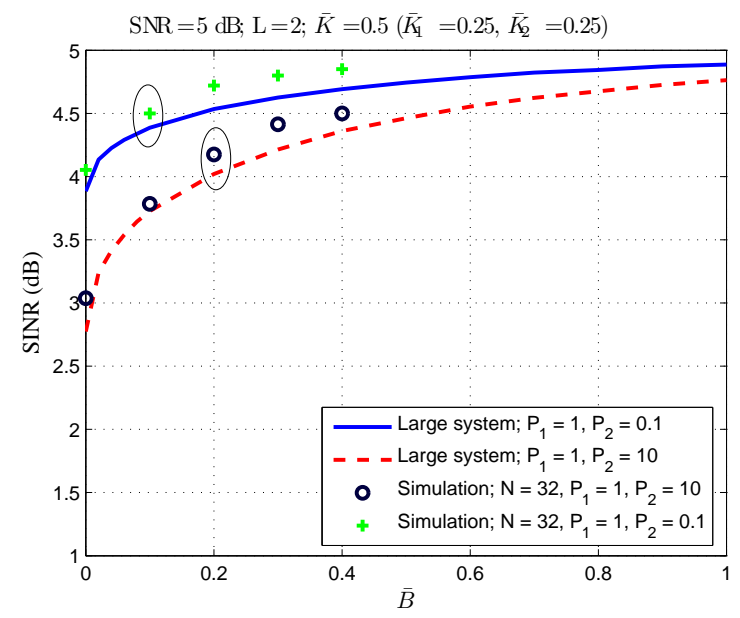

Fig. 4. Large system SINR for MMSE receiver and multipath fading with two groups of users with simulation results. SNR $=5 \mathrm{~dB}$, number of paths $L=2$ for all users, and $\bar{K}=0.5$.

where normalized loads $\bar{K}_{1}=K_{1} / N$ and $\bar{K}_{2}=K_{2} / N$. Both the large system and corresponding simulated results with $\bar{K}_{1}=\bar{K}_{2}=0.25$ and different sets of $P_{1}$ and $P_{2}$ are shown in Fig. 4 The large system performance approximates closely the performance of the system with $N=32$. As $N$ grows, the performance of a finite-size system will converge to that of the large system. In this example, reducing the transmit power of one group of users by $20 \mathrm{~dB}\left(P_{2}\right.$ from 10 to 0.1$)$ decreases the required feedback to achieve $0.5 \mathrm{~dB}$ away from the single-user performance by $\bar{B}=0.4$.

Consider a reverse-link channel, in which signals from users attenuate with distance. The received signal power at distance $d$ away from the transmitter is given by [23]

$$
P_{r}=P_{t} K_{p}\left(\frac{d_{0}}{d}\right)^{\tau}
$$

where $P_{t}$ is the transmitted power, $K_{p}$ is the constant that depends on antenna characteristics and average channel at-

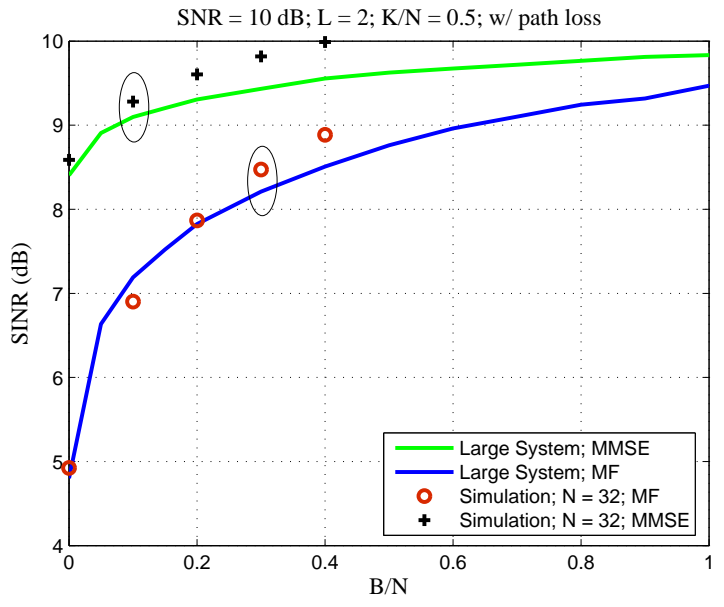

Fig. 5. A large system SINR for matched filter and MMSE receiver with multipath fading and path loss with simulation results. SNR $=10 \mathrm{~dB}$, number of paths $L=2$ for all users, $\bar{K}=0.5$, path-loss exponent $\tau=2$.

tenuation, $d_{0}$ is a reference distance, and $\tau$ is the pathloss exponent. We assume that interfering users are placed uniformly in a circular cell with distance $d_{k}$ away from the base station, where $d_{0} \leq d_{k} \leq d_{\max }, \forall k \neq 1$ and that interfering users are transmitting with the same power $P_{t}$. With the path-loss exponent $\tau=2$, a probability density for received power of interfering users at the base station is given by

$$
f_{\boldsymbol{A}_{1}}(a)=\frac{d_{0}^{2}}{K_{p} P_{t}\left(d_{\max }^{2}-d_{0}^{2}\right)}\left(\frac{K_{p} P_{t}}{a}\right)^{2}
$$

where

$$
K_{p} P_{t}\left(\frac{d_{0}}{d_{\max }}\right)^{2} \leq a \leq K_{p} P_{t} .
$$

We also assume that all users experience 2-path fading with combined gain $\alpha_{k}=1, \forall k$. Thus, $f_{\tilde{\boldsymbol{A}}_{1}}=f_{\boldsymbol{A}_{1}}$. Substituting (67) in (24) and (22) gives

$$
\eta_{\tilde{\boldsymbol{A}}_{1}}(x)=1-\frac{d_{0}^{2} K_{p} P_{t}}{d_{\max }^{2}-d_{0}^{2}} x \log \left(\frac{x+\frac{d_{\max }^{2}}{K_{p} P_{t} d_{0}^{2}}}{x+\frac{1}{K_{p} P_{t}}}\right)
$$

and

$$
\begin{aligned}
\nu_{\tilde{\boldsymbol{A}}_{1}}(x)= & \frac{d_{0}^{2} K_{p} P_{t}}{d_{\max }^{2}-d_{0}^{2}}\left[\frac{d_{\max }^{2}}{K_{p} P_{t} d_{0}^{2}} \log \left(1+x K_{p} P_{t} \frac{d_{0}^{2}}{d_{\max }^{2}}\right)\right. \\
& \left.-\frac{1}{K_{p} P_{t}} \log \left(1+x K_{p} P_{t}\right)\right]-\eta_{\tilde{\boldsymbol{A}}_{1}}(x)+1 .
\end{aligned}
$$

Applying Theorems 1 and 3, we obtain the large system performance for matched-filter and MMSE receivers. Fig. 5 compares the large system results shown in solid lines with simulation results shown with markers for $P_{t}=1, K_{p}=1$, $A_{1}=0.1, d_{0}=0.1, d_{\max }=1$, and $L=2$. The large system results approximate the simulation results for a system with $N=32$ well and we expect the difference between the two to narrow as the system size increases. With one feedback bit per signature element, both receivers perform close to the single-user performance. 


\section{CONCLUSIONS}

We have derived expressions for the large system SINR for RVQ with both a matched filter and linear MMSE receiver. The SINR is a function of a normalized load (number of users per degree of freedom) and a normalized feedback bit (number of feedback bits per degree of freedom). Both the AWGN channel with no fading and the multipath fading channel with arbitrary transmit power allocation were considered. The SINR of the quantized signature for both receivers increased approximately exponentially with $\bar{B}$. For a small load, RVQ achieved close to the single-user performance with only a fraction of the feedback bit per quantized signature coefficient. The performance of the MMSE receiver was compared with that of a matched filter derived in [9] and it was shown that the performance gap was large for a small $\bar{B}$. The simpler matched filter requires more feedback to achieve a target SINR than the MMSE receiver does.

This work assumed that the receiver could estimate the channel and interference covariances perfectly. In practice, a very accurate channel estimation is achieved by a large amount of training. How the performance of RVQ is affected by an imperfect channel estimate at the receiver (or limited training) was studied by [24]. This present work considered signature quantization for a single user. Future work may include performance analysis of a group of users with RVQquantized signatures.

\section{APPENDIX}

Proof of Corollary 2

We rewrite (48) as follows

$$
\begin{aligned}
\Phi & \left(\rho, \beta_{\text {rvq }}^{\infty}\right) \\
= & \int \log \left(1+\rho\left(\beta_{\text {rvq }}^{\infty}-\frac{1}{x+\sigma_{n}^{2}}\right)\right) f_{\boldsymbol{S}_{1} \boldsymbol{S}_{1}^{\dagger}}(x) \mathrm{d} x \\
= & \int \log \left(x+\sigma_{n}^{2}+\rho\left(\beta_{\text {rvq }}^{\infty}\left(x+\sigma_{n}^{2}\right)-1\right)\right) f_{\boldsymbol{S}_{1} \boldsymbol{S}_{1}^{\dagger}}(x) \mathrm{d} x \\
& -\int \log \left(x+\sigma_{n}^{2}\right) f_{\boldsymbol{S}_{1} \boldsymbol{S}_{1}^{\dagger}}(x) \mathrm{d} x
\end{aligned}
$$

where

$$
f_{\boldsymbol{S}_{1} \boldsymbol{S}_{1}^{\dagger}}(x)=\frac{\sqrt{(x-a)(b-x)}}{2 \pi x} \quad \text { for } \quad a \leq x \leq b,
$$

where $a=(1-\sqrt{\bar{K}})^{2}$ and $b=(1+\sqrt{\bar{K}})^{2}$ for $\bar{K}>1$.

To determine $\rho^{*}$, we take the first derivative of (72) with respect to $\rho$ given by

$$
\begin{aligned}
\frac{\mathrm{d} \Phi\left(\rho, \beta_{\mathrm{rvq}}^{\infty}\right)}{\mathrm{d} \rho}= & \frac{1}{\rho}-\frac{1}{\rho\left(\rho \beta_{\mathrm{rvq}}^{\infty}+1\right)} \\
& -\frac{1}{\left(\rho \beta_{\mathrm{rvq}}^{\infty}+1\right)^{2}} \underbrace{\int \frac{1}{x-y} f_{\boldsymbol{S}_{1} \boldsymbol{S}_{1}^{\dagger}}(x) \mathrm{d} x}_{\mathcal{S}_{f}(y)}
\end{aligned}
$$

where $\mathcal{S}_{f}(\cdot)$ is the Stieltjes transform of $f_{\boldsymbol{S}_{1} \boldsymbol{S}_{1}^{\dagger}}(\cdot)$ and

$$
y \triangleq \frac{\rho}{\rho \beta_{\mathrm{rvq}}^{\infty}+1}-\sigma_{n}^{2} .
$$

We solve for $\rho^{*}$ (or equivalently $y^{*}$ ) by setting (74) to zero and obtain

$$
\mathcal{S}_{f}\left(y^{*}\right)=\left(\rho^{*} \beta_{\mathrm{rvq}}^{\infty}+1\right) \beta_{\mathrm{rvq}}^{\infty} .
$$

Substituting the Stieltjes transform of $f_{S_{1} S_{1}^{\dagger}}(\cdot)$ and using the change of variable from (75) in (76) give

$$
\begin{array}{r}
\frac{-1+\bar{K}-y^{*} \pm \sqrt{\left(y^{*}\right)^{2}-2(\bar{K}+1) y^{*}+(\bar{K}-1)^{2}}}{2 y^{*}} \\
=\frac{\beta_{\mathrm{rvq}}^{\infty}}{1-\beta_{\mathrm{rvq}}^{\infty}\left(y^{*}+\sigma_{n}^{2}\right)} .
\end{array}
$$

Simplifying (77) gives

$$
y^{*}=\frac{\left(1-\beta_{\mathrm{rvq}}^{\infty}\left(\bar{K}-1+\sigma_{n}^{2}\right)\right)\left(1-\beta_{\mathrm{rvq}}^{\infty} \sigma_{n}^{2}\right)}{\beta_{\mathrm{rvq}}^{\infty}\left(1-\beta_{\mathrm{rvq}}^{\infty}\left(\bar{K}+\sigma_{n}^{2}\right)\right)} .
$$

With a change of variable (75), we obtain

$$
\rho^{*}=\frac{\bar{K}}{\beta_{\mathrm{rvq}}^{\infty}\left(1-\beta_{\mathrm{rvq}}^{\infty} \sigma_{n}^{2}\right)}-\frac{1}{\left(\beta_{\mathrm{rvq}}^{\infty}\right)^{2}}-\frac{1}{\beta_{\mathrm{rvq}}^{\infty}} .
$$

To show that $\rho^{*}$ achieves the maximum, we prove that $\Phi\left(\rho, \beta_{\text {rvg }}^{\infty}\right)$ is concave down when $\rho=\rho^{*}$ by computing the second derivative of $\Phi\left(\rho, \beta_{\mathrm{rvq}}^{\infty}\right)$ in $(72)$ with respect to $\rho$

$$
\begin{aligned}
& \frac{\mathrm{d}^{2} \Phi\left(\rho, \beta_{\mathrm{rvq}}^{\infty}\right)}{\mathrm{d} \rho^{2}} \\
& =-\int_{a}^{b} \frac{\left(\beta_{\mathrm{rvq}}^{\infty}\left(x+\sigma_{n}^{2}\right)-1\right)^{2}}{\left(x+\sigma_{n}^{2}+\rho\left(\beta_{\mathrm{rvq}}^{\infty}\left(x+\sigma_{n}^{2}\right)-1\right)\right)^{2}} f_{\boldsymbol{S}_{1} \boldsymbol{S}_{1}^{\dagger}}(x) \mathrm{d} x
\end{aligned}
$$$$
\leq 0 \text {. }
$$

For large enough $\beta_{\mathrm{rvq}}^{\infty} \geq \beta_{\mathrm{rvq}}^{\infty}, \rho^{*}$ in (79) can exceed $1 /\left(\beta_{\max }^{\infty}-\beta_{\mathrm{rvq}}^{\infty}\right)$. To determine $\beta_{\mathrm{rvq}}^{\infty}{ }^{*}$, we set

$$
\frac{\bar{K}}{\beta_{\mathrm{rvq}}^{\infty}\left(1-\beta_{\mathrm{rvq}}^{\infty} \sigma_{n}^{2}\right)}-\frac{1}{\left(\beta_{\mathrm{rvq}}^{\infty}\right)^{2}}-\frac{1}{\beta_{\mathrm{rvq}}^{\infty}}=\frac{1}{\beta_{\max }^{\infty}-\beta_{\mathrm{rvq}}^{\infty}} .
$$

Simplifying (82) gives the following quadratic equation

$\left[\bar{K}+\sigma_{n}^{2}-\bar{\beta} \sigma_{n}^{2}\right] \beta_{\mathrm{rvq}}^{\infty}+\left[\left(1-\bar{K}-\sigma_{n}^{2}\right) \bar{\beta}-1\right] \beta_{\mathrm{rvq}}^{\infty}+\beta_{\max }^{\infty}=0$.

Solving (83) gives the only solution

$$
\beta_{\mathrm{rvq}}^{\infty}=\frac{\bar{K}-\sqrt{\bar{K}}+\sigma_{n}^{2}}{(\bar{K}-\sqrt{\bar{K}})^{2}+2 \sigma_{n}^{2}(\bar{K}-\sqrt{\bar{K}})+\sigma_{n}^{4}} .
$$

Thus,

$$
\rho^{*}=\left\{\begin{array}{ll}
\frac{\bar{K}}{\beta_{\mathrm{rvq}}^{\infty}\left(1-\beta_{\mathrm{rvq}}^{\infty} \sigma_{n}^{2}\right)}-\frac{1}{\left(\beta_{\mathrm{rvq}}^{\infty}\right)^{2}}-\frac{1}{\beta_{\mathrm{rvq}}^{\infty},}, \quad \bar{\beta} \leq \beta_{\mathrm{rvq}}^{\infty} \leq \beta_{\mathrm{rvq}}^{\infty} * \\
\frac{\beta_{\mathrm{rvq}}^{\infty}>\beta_{\mathrm{rvq}}^{\infty} * \beta_{\mathrm{rqq}}^{\infty}}{\beta_{\max }^{\infty}},
\end{array} .\right.
$$

Substituting $\rho=\rho^{*}$ in (72) and rearranging the equation give

$$
\begin{aligned}
& \Phi\left(\rho^{*}, \beta_{\mathrm{rvq}}^{\infty}\right)=\log \left(\rho^{*} \beta_{\mathrm{rvq}}^{\infty}+1\right) \\
&+\int_{a}^{b} \log (x+\left.\sigma_{n}^{2}-\frac{\rho^{*}}{\rho^{*} \beta_{\mathrm{rvq}}^{\infty}+1}\right) f_{\boldsymbol{S}_{1} \boldsymbol{S}_{1}^{\dagger}}(x) \mathrm{d} x \\
& \quad-\int_{a}^{b} \log \left(x+\sigma_{n}^{2}\right) f_{\boldsymbol{S}_{1} \boldsymbol{S}_{1}^{\dagger}}(x) \mathrm{d} x .
\end{aligned}
$$


First, we consider the case where $\bar{\beta} \leq \beta_{\mathrm{rvq}}^{\infty} \leq \beta_{\mathrm{rvq}}^{\infty}$. Substituting $\rho^{*}$ into the first term in (86) gives

$$
\log \left(\rho^{*} \beta_{\mathrm{rvq}}^{\infty}+1\right)=\log \left(\frac{\bar{K}}{1-\beta_{\mathrm{rvq}}^{\infty} \sigma_{n}^{2}}-\frac{1}{\beta_{\mathrm{rvq}}^{\infty}}\right) .
$$

To evaluate the two integrals in (86), we apply the following lemma.

Lemma 1 (Eqs. (6)-(8) in [25]): For $\bar{K} \geq 1$,

$$
\begin{aligned}
& \int_{a}^{b} \log (x+\alpha) f_{\boldsymbol{S}_{1} \boldsymbol{S}_{1}^{\dagger}}(x) \mathrm{d} x \\
& \quad=\log (w(\alpha))-(\bar{K}-1) \log \left(1-\frac{1}{\bar{K}} v(\alpha)\right)-v(\alpha)
\end{aligned}
$$

where

$$
\begin{aligned}
& w(\alpha)=\frac{1}{2}\left(1+\bar{K}+\alpha+\sqrt{(1+\bar{K}+\alpha)^{2}-4 \bar{K}}\right), \\
& v(\alpha)=\frac{1}{2}\left(1+\bar{K}+\alpha-\sqrt{(1+\bar{K}+\alpha)^{2}-4 \bar{K}}\right) .
\end{aligned}
$$

Using Lemma 1 and (87), we can evaluate (86) for $\bar{\beta} \leq$ $\beta_{\mathrm{rvq}}^{\infty} \leq \beta_{\mathrm{rvq}}^{\infty}$,

$$
\begin{array}{r}
\Phi\left(\rho^{*}, \beta_{\text {rvq }}^{\infty}\right)=\log \left(\frac{\bar{K}}{1-\beta_{\text {rvq }}^{\infty} \sigma_{n}^{2}}-\frac{1}{\beta_{\text {rvq }}^{\infty}}\right)+\log \left(\frac{w(p)}{w\left(\sigma_{n}^{2}\right)}\right) \\
-(\bar{K}-1) \log \left(\frac{\bar{K}-v(p)}{\bar{K}-v\left(\sigma_{n}^{2}\right)}\right)-v(p)+v\left(\sigma_{n}^{2}\right)
\end{array}
$$

where

$$
p=\frac{1-\beta_{\mathrm{rvq}}^{\infty} \sigma_{n}^{2}}{\bar{K} \beta_{\mathrm{rvq}}^{\infty}-1+\beta_{\mathrm{rvq}}^{\infty} \sigma_{n}^{2}}-\frac{1}{\beta_{\mathrm{rvq}}^{\infty}}+\sigma_{n}^{2} .
$$

Next, we evaluate $\Phi\left(\rho^{*}, \beta_{\mathrm{rvq}}^{\infty}\right)$ for $\beta_{\mathrm{rvq}}^{\infty}>\beta_{\mathrm{rvq}}^{\infty}$. Substituting the value of $\rho^{*}$ from (85) gives

$$
\log \left(\rho^{*} \beta_{\mathrm{rvq}}^{\infty}+1\right)=\log \left(\beta_{\mathrm{max}}^{\infty}\right)-\log \left(\beta_{\max }^{\infty}-\beta_{\mathrm{rvq}}^{\infty}\right)
$$

and

$$
\sigma_{n}^{2}-\frac{\rho^{*}}{\rho^{*} \beta_{\mathrm{rvq}}^{\infty}+1}=\sigma_{n}^{2}-\frac{1}{\beta_{\max }^{\infty}}=-(1-\sqrt{\bar{K}})^{2} .
$$

Substituting (94) into the second term in (86) and applying Lemma 1 gives

$$
\begin{aligned}
& \int_{a}^{b} \log \left(x+\sigma_{n}^{2}-\frac{\rho^{*}}{\rho^{*} \beta_{\mathrm{rvq}}^{\infty}+1}\right) f_{\boldsymbol{S}_{1} \boldsymbol{S}_{1}^{\dagger}}(x) \mathrm{d} x \\
& \quad=\frac{1}{2} \bar{K} \log (\bar{K})-(\bar{K}-1) \log (\sqrt{\bar{K}}-1)-\sqrt{\bar{K}} .
\end{aligned}
$$

Thus, for $\beta_{\mathrm{rvq}}^{\infty}>\beta_{\mathrm{rvq}}^{\infty}$,

$$
\begin{aligned}
& \Phi\left(\rho^{*}, \beta_{\text {rvq }}^{\infty}\right)=\log \left(\beta_{\text {max }}^{\infty}\right)-\log \left(\beta_{\text {max }}^{\infty}-\beta_{\text {rvq }}^{\infty}\right) \\
& \quad+\frac{1}{2} \bar{K} \log (\bar{K})-(\bar{K}-1) \log (\sqrt{\bar{K}}-1)-\sqrt{\bar{K}} \\
& -\log \left(w\left(\sigma_{n}^{2}\right)\right)+(\bar{K}-1) \log \left(1-\frac{1}{\bar{K}} v\left(\sigma_{n}^{2}\right)\right)+v\left(\sigma_{n}^{2}\right) .
\end{aligned}
$$

Also, $\Phi\left(\rho^{*}, \beta_{\mathrm{rvq}}^{\infty}\right)=\bar{B} \log (2)$. We can explicitly solve for $\beta_{\mathrm{rvq}}^{\infty}$ as follows

$$
\begin{aligned}
\beta_{\text {rvq }}^{\infty}=\beta_{\max }^{\infty}(1- & 2^{-\bar{B}}\left[\operatorname { e x p } \left\{\frac{1}{2} \bar{K} \log (\bar{K})\right.\right. \\
- & (\bar{K}-1) \log \left(\frac{\bar{K} \sqrt{\bar{K}}-\bar{K}}{\bar{K}-v\left(\sigma_{n}^{2}\right)}\right) \\
& \left.\left.\left.\quad \log \left(w\left(\sigma_{n}^{2}\right)\right)+v\left(\sigma_{n}^{2}\right)-\sqrt{\bar{K}}\right\}\right]\right) .
\end{aligned}
$$

To solve $\bar{B}^{*}$, which corresponds to $\beta_{\mathrm{rvq}}^{\infty}$ (84), we substitute $\beta_{\mathrm{rvq}}^{\infty *}$ in (97).

\section{REFERENCES}

[1] P. Rapajic and B. Vucetic, "Linear adaptive transmitter-receiver structures for asynchronous CDMA systems," European Trans. on Telecommun., vol. 6, no. 1, pp. 21-28, Jan-Feb 1995.

[2] S. Ulukus and R. D. Yates, "Iterative construction of optimum sequence sets in synchronous CDMA systems," IEEE Trans. Inf. Theory, vol. 47, no. 5, pp. 1989-1998, Jul. 2001.

[3] T. F. Wong and T. M. Lok, "Transmitter adaptation in multicode DSCDMA systems," IEEE J. Sel. Areas Commun., vol. 19, no. 1, pp. 69-82, Jan. 2001.

[4] G. S. Rajappan and M. L. Honig, "Signature sequence adaptation for DS-CDMA with multipath," IEEE J. Sel. Areas Commun., vol. 20, no. 2, pp. 384-395, Feb. 2002.

[5] P. Viswanath, V. Anantharam, and D. N. C. Tse, "Optimal sequences, power control and capacity of spread-spectrum systems with multiuser linear receivers," IEEE Trans. Inf. Theory, vol. 45, no. 6, pp. 1968-1983, Sep. 1999.

[6] - "Optimal sequences and sum capacity of synchronous CDMA systems," IEEE Trans. Inf. Theory, vol. 45, no. 6, pp. 1984-1991, Sep. 1999.

[7] C. Rose, S. Ulukus, and R. D. Yates, "Wireless systems and interference avoidance," IEEE Trans. Wireless Commun., vol. 1, no. 3, pp. 415-428, Jul. 2002.

[8] W. Santipach and M. L. Honig, "Signature optimization for CDMA with limited feedback," IEEE Trans. Inf. Theory, vol. 51, no. 10, pp. 34753492, Oct. 2005.

[9] W. Dai, Y. Liu, and B. Rider, "The effect of finite rate feedback on CDMA signature optimization and MIMO beamforming vector selection," IEEE Trans. Inf. Theory, vol. 55, no. 8, pp. 3651-3669, Aug. 2009.

[10] D. J. Love and R. W. Heath, Jr., "Grassmannian beamforming for multiple-input multiple-output wireless systems," IEEE Trans. Inf. Theory, vol. 49, no. 10 , pp. 2735-2745, Oct. 2003.

[11] K. K. Mukkavilli, A. Sabharwal, E. Erkip, and B. Aazhang, "On beamforming with finite rate feedback in multiple antenna systems," IEEE Trans. Inf. Theory, vol. 49, no. 10, pp. 2562-2579, Oct. 2003.

[12] W. Santipach and M. L. Honig, "Capacity of a multiple-antenna fading channel with a quantized precoding matrix," IEEE Trans. Inf. Theory, vol. 55, no. 3, pp. 1218-1234, Mar. 2009.

[13] J. C. Roh and B. D. Rao, "Transmit beamforming in multiple-antenna systems with finite rate feedback: A VQ-based approach," IEEE Trans. Inf. Theory, vol. 52, no. 3, pp. 1101-1112, Mar. 2006.

[14] V. K. N. Lau, Y. Liu, and T.-A. Chen, "On the design of MIMO blockfading channels with feedback-link capacity constraint," IEEE Trans. Commun., vol. 52, no. 1, pp. 62-70, Jan. 2004.

[15] S. Zhou, Z. Wang, and G. B. Giannakis, "Performance analysis for transmit-beamforming with finite-rate feedback," in Proc. Conf. on Inform. Sciences and Systems, Princeton, NJ, Mar. 2004, pp. 880-885.

[16] U. Madhow and M. L. Honig, "MMSE interference suppression for direct-sequence spread-spectrum CDMA," IEEE Trans. Commun., vol. 42, no. 12, pp. 3178-3188, Dec. 1994.

[17] A. Narula, M. J. Lopez, M. D. Trott, and G. W. Wornell, "Efficient use of side information in multiple antenna data transmission over fading channels," IEEE J. Sel. Areas Commun., vol. 16, no. 8, pp. 1423-1436, Oct. 1998

[18] D. J. Love, R. W. Heath, Jr., W. Santipach, and M. L. Honig, "What is the value of limited feedback for MIMO channels?" IEEE Commun. Mag., vol. 42, no. 10 , pp. 54-59, Oct. 2004.

[19] J. Galambos, The Asymptotic Theory of Extreme Order Statistics, 2nd ed. Robert E. Krieger, 1987.

[20] A. M. Tulino and S. Verdú, "Random matrix theory and wireless communications," Foundations and Trends in Communications and Information Theory, vol. 1, no. 1, pp. 1-182, 2004.

[21] V. A. Marčenko and L. A. Pastur, "Distribution of eigenvalues for some sets of random matrices," Math. USSR-Sbornik, vol. 1, pp. 457-483, 1967.

[22] R. Gray, "On the asymptotic eigenvalue distribution of Toeplitz matrices," IEEE Trans. Inf. Theory, vol. 18, no. 6, pp. 725 - 730, Nov. 1972.

[23] T. S. Rappaport, Wireless Communications - Principles and Practice, 2nd ed. Englewood Cliffs, NJ: Prentice-Hall, 2001. 
IEEE TRANSACTIONS ON COMMUNICATIONS

[24] W. Santipach and M. L. Honig, "Optimization of training and feedback overhead for beamforming over block fading channels," IEEE Trans. Inf. Theory, vol. 56, no. 12, pp. 6103-6115, Dec. 2010.

[25] P. B. Rapajic and D. Popescu, "Information capacity of a random signature multiple-input multiple-output channel," IEEE Trans. Commun., vol. 48 , no. 8, pp. 1245-1248, Aug. 2000. 